\title{
A geometric approach to sector decomposition
}

\section{Takahiro Ueda*}

Graduate School of Pure and Applied Sciences, University of Tsukuba, 1-1-1 Tennodai, Tsukuba, Ibaraki 305-8571, Japan

E-mail: tueda@het.ph.tsukuba.ac.jp

\section{Toshiaki Kaneko}

Computing Research Center, High Energy Accelerator Research Organization (KEK),

1-1 Oho, Tsukuba, Ibaraki 305-0801, Japan

E-mail: toshiaki.kaneko@kek.jp

We present a non-iterative method of sector decomposition, which is used to factorise overlapping singularities from multi-dimensional integrals appearing in perturbative quantum field theory. The problem is converted to a set of ones in convex geometry, and they are solved with algorithms developed in computational geometry. A test implementation shows that this method produces less number of decomposed sectors than usual iterative sector decompositions.

3rd Computational Particle Physics Workshop

September 23-25, 2010

KEK Tsukuba Japan

\footnotetext{
${ }^{*}$ Speaker.
} 


\section{Introduction}

Recent high-precision experiments in high energy physics require high-precision theoretical predictions in perturbation theory for a wide variety of physical processes, and the automatisation of theoretical calculations are indispensable. In the evaluation of Feynman amplitudes with massless particles, infrared (soft and collinear) divergences can appear in each amplitude separately. However, for suitably defined physical observables, they cancel out among virtual loop corrections and real emission processes. One has to regulate infrared divergences at intermediate calculation steps.

In perturbative $\mathrm{QCD}$, it is common that the divergences are regulated by dimensional regularisation, and divergent parts are expressed as poles in terms of $\varepsilon=(4-D) / 2$, where $D$ is the space-time dimension. An one-dimensional example of an isolation of the divergence is as follows:

$$
\begin{aligned}
\int_{0}^{1} d x \frac{f(x)}{x^{1-\varepsilon}} & =\int_{0}^{1} d x \frac{f(0)}{x^{1-\varepsilon}}+\int_{0}^{1} d x \frac{f(x)-f(0)}{x^{1-\varepsilon}} \\
& =\frac{f(0)}{\varepsilon}+\sum_{n=0}^{\infty} \frac{\varepsilon^{n}}{n !} \int_{0}^{1} d x \ln ^{n}(x) \frac{f(x)-f(0)}{x},
\end{aligned}
$$

where we have assumed that $f(x)$ is a finite function in the domain of integration. This integral is divergent for $\varepsilon=0$ due to the factor $1 / x^{1-\varepsilon}$, which produces a logarithmic divergence by integration around $x \sim 0$. In the right-hand side, a subtraction technique was used to isolate a pole of $\varepsilon$. The integral in the first term can be analytically performed, and the second term becomes finite for $\varepsilon \rightarrow 0$. This example shows that, if the singular part of the integrand is factored out as powers of an integration variable, one can easily extract divergent part as poles in terms of $\varepsilon$.

The extraction of divergences from more complicated integrals with overlapping divergences can be performed by using sector decomposition [1] in an algorithmic fashion [2-4]. Let us consider the following two-dimensional example:

$$
I=\int_{0}^{1} d x \int_{0}^{1} d y \frac{f(x, y)}{(x+y)^{2-\varepsilon}}
$$

with a finite function $f(x, y)$ for the domain of integration. This integral is divergent for $\varepsilon=0$ due to a singularity around both $x, y \sim 0$. We divide the integration region such that $x$ and $y$ are ordered in each region (sector):

$$
I=\int_{0}^{1} d x \int_{0}^{1} d y \frac{f(x, y)}{(x+y)^{2-\varepsilon}}[\Theta(x>y)+\Theta(y>x)] .
$$

In the sector with $x>y$, the singular behaviour is controlled by the dominant term $x$ in the denominator. We rescale $y \rightarrow x y$ and then we factor out $x$ from the denominator. In the sector with $y>x$, on the other hand, we factor out $y$ with a rescaling $x \rightarrow x y$. We then obtain

$$
I=\int_{0}^{1} d x \int_{0}^{1} d y \frac{f(x, x y)}{x^{1-\varepsilon}(1+y)^{2-\varepsilon}}+\int_{0}^{1} d x \int_{0}^{1} d y \frac{f(x y, y)}{y^{1-\varepsilon}(1+x)^{2-\varepsilon}} .
$$

Now we can extract the poles from each integral by the subtraction technique.

For more complicated integrands, usual sector decomposition method divides the integration region and find appropriate new variables by an iterative way, but it is not trivial whether the 
iteration always terminates or not. Actually it was found that a simple iterative method may fail into an infinite loop. To avoid this problem, strategies for iterated sector decomposition which are guaranteed to terminate mathematically have been proposed by several authors [5-7]. Since one has to compute the integration for each sector, the sector decomposition method is desired to produce as less number of sectors as possible. Up to now, several practical packages, in which iterated sector decomposition is implemented, are publicly available [5, 6, 8 .

We propose another method of sector decomposition, which is based on a classification of asymptotic behaviour of polynomials around the origin. We recast the problem to a set of ones in convex geometry, which can be solved with algorithms developed in computational geometry. These are deterministic algorithms without iterations. In this talk, based on Ref. [10], we present our basic ideas and show results from a test implementation of this method.

\section{Factorisation of overlapping singularities}

Let us consider the following polynomial:

$$
Q\left(x_{1}, x_{2}\right)=x_{1} x_{2}^{4}+x_{1}^{2} x_{2}^{2}+x_{1}^{4} x_{2} .
$$

The our goal is to perform sector decomposition such as

$$
\int_{0}^{1} d^{2} x\left[Q\left(x_{1}, x_{2}\right)\right]^{\beta}=\int_{0}^{1} d^{2} x\left(x_{1} x_{2}^{4}+x_{1}^{2} x_{2}^{2}+x_{1}^{4} x_{2}\right)^{\beta}=\sum \int_{0}^{1} d^{2} x x_{1}^{\alpha_{1}} x_{2}^{\alpha_{2}}\left[1+\tilde{Q}\left(x_{1}, x_{2}\right)\right]^{\beta},
$$

where $\tilde{Q}$ is a polynomial which vanishes at $x_{1}=x_{2}=0$, and then the polynomial $1+\tilde{Q}$ in the square bracket has a finite value at the origin.

First, we split the integration region into three sectors such that in each sector one of the terms in $Q$ is dominant over other terms:

$$
\begin{aligned}
I=\int_{0}^{1} d^{2} x\left(x_{1} x_{2}^{4}+\right. & \left.x_{1}^{2} x_{2}^{2}+x_{1}^{4} x_{2}\right)^{\beta}=\int_{0}^{1} d^{2} x\left(x_{1} x_{2}^{4}+x_{1}^{2} x_{2}^{2}+x_{1}^{4} x_{2}\right)^{\beta} \\
& \times\left[\Theta\left(x_{1} x_{2}^{4}>x_{1}^{2} x_{2}^{2}, x_{1}^{4} x_{2}\right)+\Theta\left(x_{1}^{2} x_{2}^{2}>x_{1} x_{2}^{4}, x_{1}^{4} x_{2}\right)+\Theta\left(x_{1}^{4} x_{2}>x_{1} x_{2}^{4}, x_{1}^{2} x_{2}^{2}\right)\right] .
\end{aligned}
$$

In the sector with $x_{1} x_{2}^{4}>x_{1}^{2} x_{2}^{2}, x_{1}^{4} x_{2}$, the singular behaviour at the origin is governed by the dominant term $x_{1} x_{2}^{4}$. Thus we factor out $x_{1} x_{2}^{4}$, and the rest of the terms has a finite value by construction:

$$
\begin{aligned}
I_{1} & =\int_{0}^{1} d^{2} x\left(x_{1} x_{2}^{4}+x_{1}^{2} x_{2}^{2}+x_{1}^{4} x_{2}\right)^{\beta} \Theta\left(x_{1} x_{2}^{4}>x_{1}^{2} x_{2}^{2}, x_{1}^{4} x_{2}\right) \\
& =\int_{0}^{1} d^{2} x\left(x_{1} x_{2}^{4}\right)^{\beta}\left(1+\frac{x_{1}^{2} x_{2}^{2}}{x_{1} x_{2}^{4}}+\frac{x_{1}^{4} x_{2}}{x_{1} x_{2}^{4}}\right)^{\beta} \Theta\left(x_{1} x_{2}^{4}>x_{1}^{2} x_{2}^{2}, x_{1}^{4} x_{2}\right) \\
& =\int_{0}^{1} d^{2} x x_{1}^{\beta} x_{2}^{4 \beta}\left(1+\frac{x_{1}}{x_{2}^{2}}+\frac{x_{1}^{3}}{x_{2}^{3}}\right)^{\beta} \Theta\left(x_{2}^{2}>x_{1}\right) .
\end{aligned}
$$

In this form, the singular part of the integrand is factored out as powers of integration variables, but the integration region is not a unit square and the terms in the square bracket are not monomials. These difficulties can be removed with a rescaling $x_{1} \rightarrow x_{1} x_{2}^{2}$. We then obtain

$$
I_{1}=\int_{0}^{1} d^{2} x x_{1}^{\beta} x_{2}^{6 \beta+2}\left(1+x_{1}+x_{1}^{3} x_{2}^{3}\right)^{\beta}
$$


One can factor out dominant terms in the other two sectors in a similar way, and rescale $\left(x_{1}, x_{2}\right) \rightarrow$ $\left(x_{1} x_{2}^{2}, x_{1}^{2} x_{2}\right)$ and $x_{2} \rightarrow x_{1}^{2} x_{2}$, respectively, to obtain

$$
\begin{aligned}
I= & \int_{0}^{1} d^{2} x x_{1}^{\beta} x_{2}^{6 \beta+2}\left(1+x_{1}+x_{1}^{3} x_{2}^{3}\right)^{\beta}+3 \int_{0}^{1} d^{2} x x_{1}^{6 \beta+2} x_{2}^{6 \beta+2}\left(1+x_{1}^{3}+x_{2}^{3}\right)^{\beta} \\
& +\int_{0}^{1} d^{2} x x_{1}^{6 \beta+2} x_{2}^{\beta}\left(1+x_{2}+x_{1}^{3} x_{2}^{3}\right)^{\beta} .
\end{aligned}
$$

Now one can easily isolate poles by the subtraction technique.

The above example shows that sector decomposition can be solved by the following two steps:

1. To determine the integration regions where each term becomes dominant and split sectors by them. Then the dominant term can be factored out.

2. To find a new parametrisation of the integration variables such that the integration region is restored to a unit hypercube and other terms are expressed as monomials. In general, this may require more splitting sectors.

In the next section, we will see that these problems can be recast to ones in convex geometry.

\section{Geometric approach}

In order to find the dominant terms, we change the integration variables $x_{i}=e^{-y_{i}}$. The polynomial $Q$ becomes

$$
Q=x_{1} x_{2}^{4}+x_{1}^{2} x_{2}^{2}+x_{1}^{4} x_{2}=e^{-\left(y_{1}+4 y_{2}\right)}+e^{-\left(2 y_{1}+2 y_{2}\right)}+e^{-\left(4 y_{1}+y_{2}\right)} \equiv e^{-\vec{P}_{1} \cdot \vec{y}}+e^{-\vec{P}_{2} \cdot \vec{y}}+e^{-\vec{P}_{3} \cdot \vec{y}},
$$

with

$$
\vec{P}_{1}=(1,4), \quad \vec{P}_{2}=(2,2), \quad \vec{P}_{3}=(4,1), \quad \vec{y}=\left(y_{1}, y_{2}\right) .
$$

In this way, each monomial in $Q$ can be mapped one-to-one to a integer vector $\vec{P}_{i}$ in the twodimensional Euclidean space (Fig. 1h). For the sector where the first term is dominant, we can write

$$
I_{1}=\int_{0}^{\infty} d^{2} y e^{-\overrightarrow{1} \cdot \vec{y}}\left(e^{-\vec{P}_{1} \cdot \vec{y}}+e^{-\vec{P}_{2} \cdot \vec{y}}+e^{-\vec{P}_{3} \cdot \vec{y}}\right)^{\beta} \Theta\left(\left(\vec{P}_{2}-\vec{P}_{1}\right) \cdot \vec{y}>0\right) \Theta\left(\left(\vec{P}_{3}-\vec{P}_{1}\right) \cdot \vec{y}>0\right),
$$

where $\overrightarrow{1}=(1,1)$. The integration region is a convex polyhedral cone (with the apex at the origin). We denote the region where the first term is dominant as $\Delta_{1}$ (Fig. 1b):

$$
\Delta_{1}=\left\{\vec{y} \in \mathbb{R}_{\geq 0}^{2} \mid\left(\vec{P}_{2}-\vec{P}_{1}\right) \cdot \vec{y}>0 \wedge\left(\vec{P}_{3}-\vec{P}_{1}\right) \cdot \vec{y}>0\right\},
$$

and factor out the dominant term to obtain

$$
I_{1}=\int_{0}^{\infty} d^{2} y e^{-\left(\beta \vec{P}_{1}+\overrightarrow{1}\right) \cdot \vec{y}}\left(1+e^{-\left(\vec{P}_{2}-\vec{P}_{1}\right) \cdot \vec{y}}+e^{-\left(\vec{P}_{3}-\vec{P}_{1}\right) \cdot \vec{y}}\right)^{\beta} \Theta\left(\vec{y} \in \Delta_{1}\right) .
$$

In general, an $n$-variable polynomial $Q$ corresponds to a set of points in $n$-dimensional Euclidean space $Z=\left(\vec{P}_{1}, \vec{P}_{2}, \ldots, \vec{P}_{m}\right)$ with $\vec{P}_{i} \in \mathbb{Z}_{\geq 0}^{n}$. Then the region where $i$-th term is dominant is 


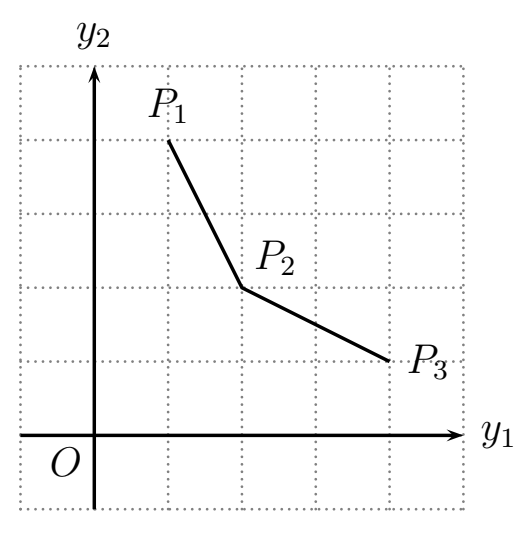

(a)

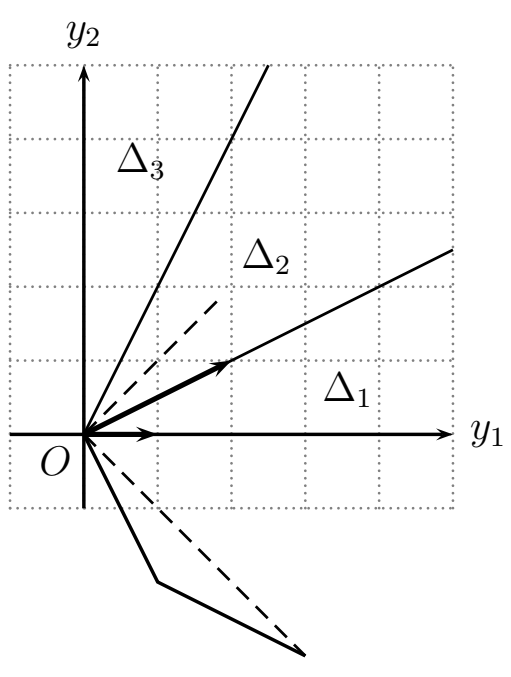

(b)

Figure 1: (a) The points in two-dimensional Euclidean space corresponding to the monomials in $Q$ and (b) the corresponding regions where each monomial is dominant.

given as $\Delta_{i}=\left\{\vec{y} \in \mathbb{R}_{\geq 0}^{n} \mid\left(\vec{P}_{j}-\vec{P}_{i}\right) \cdot \vec{y}>0, \forall \vec{P}_{j} \in Z\right\}$. One can express this region in terms of convex geometry. For a finite set $S$, a convex polyhedral cone $C$ is defined by

$$
C(S)=\left\{\sum_{\vec{v} \in S} r_{v} \vec{v} \in \mathbb{R}^{n} \mid r_{v} \geq 0, \forall \vec{v} \in S\right\}
$$

A dual cone $C^{\vee}$ of a convex polyhedral cone $C$ is defined by

$$
C(S)^{\vee}=\left\{\vec{y} \in \mathbb{R}^{n} \mid \vec{v} \cdot \vec{y} \geq 0, \forall \vec{v} \in C(S)\right\} .
$$

Then $\Delta_{i}$ can be expressed as

$$
\Delta_{i}=C\left(Z_{i}\right)^{\vee} \cap \mathbb{R}_{\geq 0}^{n}, \quad \text { with } Z_{i}=\left(\vec{P}_{1}-\vec{P}_{i}, \ldots, \vec{P}_{m}-\vec{P}_{i}\right) .
$$

If there are two or more polynomials one must consider, one should consider an intersection of dual cones, such as $\Delta_{i j}=C\left(Z_{i}\right)^{\vee} \cap C\left(Z_{j}^{\prime}\right)^{\vee} \cap \mathbb{R}_{\geq 0}^{n}$.

The integration region $\Delta_{1}$ in Eq. (3.5) is a simplicial cone, a convex polyhedral cone with $n$ edges in $n$-dimensional space. This means one can parametrise a point $\vec{y}$ in $\Delta_{1}$ by barycentric coordinate:

$$
y_{i}=\sum_{j=1}^{2}\left(\vec{v}_{j}\right)_{i} u_{j}, \quad u_{j} \in \mathbb{R}_{\geq 0},
$$

where $\vec{v}_{i}$ 's are integer vectors corresponding to edges of $\Delta_{1}$. From Fig. 1b, one can read $v_{1}=(1,0)$ and $v_{2}=(2,1)$ for $\Delta_{1}$. We subsequently change the variable from $y_{i}$ to $u_{i}$ then from $u_{j}$ to $z_{i}=e^{-u_{i}}$. We finally obtain

$$
\begin{aligned}
I_{1} & =|\operatorname{det} V| \int_{0}^{1} d^{2} z \prod_{i=1}^{2} z_{i}^{\left(\beta \vec{P}_{1}+\overrightarrow{1}\right) \cdot \vec{v}_{i}-1}\left[1+\prod_{i=1}^{2} z_{i}^{\left(\vec{P}_{2}-\vec{P}_{1}\right) \cdot \vec{v}_{i}}+\prod_{i=1}^{2} z_{i}^{\left(\vec{P}_{3}-\vec{P}_{1}\right) \cdot \vec{v}_{i}}\right]^{\beta} \\
& =\int_{0}^{1} d^{2} z z_{1}^{\beta} z_{2}^{6 \beta+2}\left(1+z_{1}+z_{1}^{3} z_{2}^{3}\right)^{\beta},
\end{aligned}
$$


Table 1: Comparison of number of sectors among different methods. The numbers in columns "A", "B", "C", "S" and "X" indicate corresponding strategy described in Refs. [5] and [6]. The numbers in column "H" are from Ref. [14. The numbers marked with "*" are given by [15]. "This method" indicates the number of sectors obtained by our method. "Exponential S.D." indicates the number of sectors before the triangulation. As described in Ref. [6], "F" means that the sector decomposition fails and "M" means that the memory overflow happened during the sector decomposition on a $8 \mathrm{~Gb}$ machine.

\begin{tabular}{|l|lllll|l|l|l|}
\hline Diagram & A & B & C & S & X & H & $\begin{array}{l}\text { This } \\
\text { method }\end{array}$ & $\begin{array}{l}\text { Exponential } \\
\text { S.D. }\end{array}$ \\
\hline Bubble & 2 & 2 & 2 & $2^{*}$ & 2 & & 2 & 2 \\
Triangle & 3 & 3 & 3 & $3^{*}$ & 3 & & 3 & 3 \\
Box & 12 & 12 & 12 & 12 & 12 & & 12 & 8 \\
\hline Tbubble & 58 & 48 & 48 & $48^{*}$ & 48 & & 48 & 36 \\
Double box, $p_{i}^{2}=0$ & 775 & 586 & 586 & 362 & 293 & 282 & 266 & 106 \\
Double box, $p_{4}^{2} \neq 0$ & $543^{*}$ & $245^{*}$ & $245^{*}$ & $230^{*}$ & $192^{*}$ & 197 & 186 & 100 \\
Double box, $p_{i}^{2}=0$ & 1138 & 698 & 698 & $441^{*}$ & 395 & & 360 & 120 \\
$\quad$ nonplanar & & & & & & & & \\
D420 & 8898 & 564 & 564 & 180 & $\mathrm{~F}$ & & 168 & 100 \\
\hline 3 loop vertex (A8) & $4617^{*}$ & $1196^{*}$ & $1196^{*}$ & $871^{*}$ & $750^{*}$ & 684 & 684 & 240 \\
Triple box & $\mathrm{M}$ & 114256 & 114256 & 22657 & 10155 & & 6568 & 856 \\
\hline
\end{tabular}

where $V$ is a matrix constructed as $V=\left(v_{1}, v_{2}\right)$. Note that $\left(\vec{P}_{i}-\vec{P}_{1}\right) \cdot \vec{v}_{j}$ is a non-negative integer since $\vec{v}_{j} \in \Delta_{1}$.

In three or higher dimension, a convex polyhedral cone may not be a simplicial cone. In a such case, we need triangulation, splitting a convex polyhedral cone into simplicial cones. The triangulation of convex polyhedral cones is not uniquely determined, and the number of sectors will depend on the algorithm.

\section{Test implementation}

We have made a test implementation of this method. For a convex hull method to construct intersections of dual cones, we adapted incremental algorithm described in Ref. [11], and modified it for convex polyhedral cones. For the triangulation of convex polyhedral cones, we used our own algorithm described in Ref. [10].

The procedure of constructing convex hull was checked by comparison with another package qhull [12]. The triangulation of convex polyhedral cones was checked by computing integration volume of resulting simplexes. As non-trivial checks, we compared integrated values of several loop diagrams in the literature. The input to our program was given by the program package partly described in Ref. [13], which builds expressions of loop integrals and performs primary sector decomposition. The output was passed to the same program package, which continues the subsequent isolation of divergences by the subtraction, expansion in terms of $\varepsilon$, and numerical integration steps. It was confirmed that integrated values agree well within the statistical errors. We show the numbers of decomposed sectors in Table 1 


\section{Conclusion}

We have proposed a new method of sector decomposition employing a geometric interpretation of the problem. The original problem is converted to a set of problems in convex geometry. A test implementation shows that the number of decomposed sectors is less than usual iterative sector decompositions.

\section{References}

[1] K. Hepp, Proof of the Bogolyubov-Parasiuk theorem on renormalization, Commun. Math. Phys. 2 (1966) 301.

[2] T. Binoth and G. Heinrich, An automatized algorithm to compute infrared divergent multi-loop integrals, Nucl. Phys. B585 (2000) 741 hep-ph/0004013.

[3] T. Binoth and G. Heinrich, Numerical evaluation of multi-loop integrals by sector decomposition, Nucl. Phys. B680 (2004) 375 hep-ph/0305234.

[4] T. Binoth and G. Heinrich, Numerical evaluation of phase space integrals by sector decomposition, Nucl. Phys. B693 (2004) 134 hep-ph/0402265.

[5] C. Bogner and S. Weinzierl, Resolution of singularities for multi-loop integrals, Comput. Phys. Commun. 178 (2008) 596 arXiv:0709.4092].

[6] A.V. Smirnov and M.N. Tentyukov, Feynman Integral Evaluation by a Sector decomposiTion Approach (FIESTA), Comput. Phys. Commun. 180 (2009) 735 arXiv:0807.4129.

[7] A.V. Smirnov and V.A. Smirnov, Hepp and Speer Sectors within Modern Strategies of Sector Decomposition, JHEP 05 (2009) 004 arXiv: 0812 .4700.

[8] A.V. Smirnov, V.A. Smirnov, and M. Tentyukov, FIESTA 2: parallelizeable multiloop numerical calculations, arXiv:0912.0158

[9] J. Carter and G. Heinrich, SecDec: A general program for sector decomposition, arXiv:1011.5493.

[10] T. Kaneko and T. Ueda, A geometric method of sector decomposition, Comput. Phys. Commun. 181 (2010) 1352 arXiv:0908.2897.

[11] H. Edelsbrunner, Algorithms in combinatorial geometry, Springer-Verlag, New York.

[12] C.B. Barber, D.P. Dobkin, and H. Huhdanpaa, The Quickhull algorithm for convex hulls, ACM Trans. Math. Softw. 22 (1996) 469. Program package qhull is available at http://www. qhull.org

[13] T. Ueda and J. Fujimoto, New implementation of the sector decomposition in FORM, PoS (ACAT08) 120 arXiv:0902.2656.

[14] G. Heinrich, Sector Decomposition, Int. J. Mod. Phys. A23 (2008) 1457 arXiv:0803.4177.

[15] A.V. Smirnov, private communication. 